\title{
Enabling Homomorphically Encrypted Inference for Large DNN Models
}

\author{
Guillermo Lloret-Talavera, Marc Jorda, Harald Servat, Fabian Boemer, Chetan Chauhan, \\ Shigeki Tomishima, Nilesh N. Shah, and Antonio J. Peña
}

\begin{abstract}
The proliferation of machine learning services in the last few years has raised data privacy concerns. Homomorphic encryption (HE) enables inference using encrypted data but it incurs 100x-10,000x memory and runtime overheads. Secure deep neural network (DNN) inference using $\mathrm{HE}$ is currently limited by computing and memory resources, with frameworks requiring hundreds of gigabytes of DRAM to evaluate small models. To overcome these limitations, in this paper we explore the feasibility of leveraging hybrid memory systems comprised of DRAM and persistent memory. In particular, we explore the recently-released Intel ${ }^{\circledR}$ Optane ${ }^{\mathrm{TM}}$ PMem technology and the Intel ${ }^{\circledR} \mathrm{HE}$-Transformer $\mathrm{nGraph}{ }^{\circledR}$ to run large neural networks such as MobileNetV2 (in its largest variant) and ResNet-50 for the first time in the literature. We present an in-depth analysis of the efficiency of the executions with different hardware and software configurations. Our results conclude that DNN inference using HE incurs on friendly access patterns for this memory configuration, yielding efficient executions.
\end{abstract}

Index Terms—Privacy-Preserving Machine Learning, Deep Learning, Homomorphic Encryption.

\section{INTRODUCTION}

$\mathrm{M}$ ACHINE learning (ML) enables solving problems that are infeasible with traditional techniques in fields like computer vision or speech recognition. Although the available computational power is ever-increasing, most ML projects are still relatively highly compute-intensive. Data scientists and commercial ML deployments often resort to third-party providers to speed up training and inference tasks. For instance, mobile-based voice recognition is frequently implemented as a cloud service.

Practical homomorphic encryption (HE) implementations emerged recently and enable computations directly on encrypted data; the (encrypted) result is correct as if it were produced by the traditional method (decryption, computation, and encryption) and may be decrypted using the secret key. $\mathrm{HE}$ is not exempt from drawbacks that render it currently impractical in many scenarios: the size of the data increases fiercely when encrypted, whereas the computation time is considerably higher than that over unencrypted data. HE requires the selection of several parameters; ensuring high security level, such as post-quantum, requires large memory and runtime overheads [1]. Values for this overhead vary depending on the security parameters, usually being in the order of 100x for compute and $100 x-10,000 x$ for data size. There are clear use cases for deep neural network (DNN) model and dataset encryption, such as intellectual property or sensitive data protection. While customary RAM, based on DRAM technology, is far from able to entirely host production-sized homomorphically encrypted DNNs or associated datasets, out-of-

- G. Lloret-Talavera, M. Jorda, and A. J. Peña are with the Barcelona Supercomputing Center (BSC).

E-mail: \{guillermo.lloret,marc.jorda,antonio.pena\}@bsc.es

- H. Servat, F. Boemer, C. Chauhan, S. Tomishima, and N. N. Shah are with Intel Corporation.

E-mail: \{harald.servat,fabian.boemer,chetan.chauhan, shigeki.tomishima, nilesh.n.shah\}@intel.com band algorithms will suffer from intrinsic data movement overheads, apart from the added code complexity. Only reduced-size homomorphically encrypted DNN inference cases have been reported, on top of DRAM, while training is considered too time-consuming [2].

Recently, dual in-line memory modules (DIMMs) based on new technologies have become commercially available. One of these alternatives to traditional DRAM is the Intel ${ }^{\circledR}$ Optane ${ }^{\mathrm{TM}}$ PMem product line [3]. Besides nonvolatility, it offers a much larger capacity than DRAM, with up to 512 GB of memory in a single DIMM. However, the access latency for the persistent memory is considerably higher than that of DRAM, especially when storing data. With respect to DRAM, its latencies increase $2 x-6 x$ for reads and $6 x-30 x$ for writes depending on the access pattern, whereas bandwidth decreases around $75 \%$ for reads and $90 \%$ for writes. To palliate this large gap with DRAM performance, which is exacerbated at non-sequential access patterns due to large access block sizes, DRAM and PMem are usually combined on a hybrid memory system. This technology may be an enabler for large DNNs to be run using HE in a single machine; however, prior to this work, it was unclear how Intel Optane PMem latency characteristics would affect performance.

In this work, we present, for the first time in the literature, an exhaustive performance analysis of an HE framework running on a system with hybrid DRAM + persistent memory subsystems. We present the results of our experiments including different DRAM capacities, but also the largest DNNs reported to date on an HE inference framework (namely MobileNetV2 on its largest variant and ResNet-50), as enabled by the use of persistent memory technology. We have analyzed the performance of Intel Optane PMem to determine its viability for this specific use case. Our results reveal that HE inference yields a friendly access pattern to Intel's implementation of persis- 
tent memory technology, hence enabling for the first time the execution of large DNN models leveraging HE.

In summary, the contributions of this article are: (1) We report the largest DNN models run to date using HE; (2) We report for the first time the use of persistent memory technology to enable large DNN inference leveraging HE; and (3) We provide the corresponding novel in-depth analysis of the viability of using persistent memory technologies for this specific use case.

The rest of the document is structured as follows. Section 2 provides the necessary background. Section 3 discusses related work in the literature. Section 4 describes the testbed used in our experiments. Section 5 presents our results and their analysis. Section 6 reviews the conclusions of this work.

\section{BACKGROUND}

This section is intended to provide readers with the necessary background information to understand the rest of the manuscript. First we introduce HE. Next, we discuss the main features of the persistent memory implementation we leveraged in this study.

\subsection{Homomorphic Encryption}

HE is a type of encryption that enables limited computation on the ciphertext, without use of the secret key. This feature allows data to remain confidential when being processed in an untrusted environment.

HE was proposed in 1978 [4]. Over the next 30 years, researchers discovered a variety of partial HE (PHE) schemes-supporting a single operation, such as addition or multiplication; somewhat HE (SHE) schemes-supporting several operations, such as both addition and multiplication, but on only a subset of circuits; and leveled HE (LHE) schemes-supporting arbitrary circuits, up to a limited size or depth. In our work, we focus on the CKKS LHE scheme [5].

The security of many HE schemes, including CKKS, is based on the ring learning with error (RLWE) problem [6], whose security derives from the hardness of the shortest vector problem (SVP) in lattices. The RLWE problem uses polynomials in $\mathcal{R}_{q}:=\mathbb{Z}_{q}[X] /\left(X^{N}+1\right)$, where the polynomial modulus $N$ is typically a power of two and the coefficient modulus $q$ is a prime number. Concretely, $\mathcal{R}_{q}$ contains polynomials of degree $N-1$ whose coefficients are integers modulo $q$. Addition and multiplication in $\mathcal{R}_{q}$ may be performed via regular polynomial addition and multiplication, followed by reduction by $X^{N}+1$, and coefficient-wise reduction modulo $q$. Given polynomials $a_{i}(x), s(x)$ drawn uniformly at random from $\mathcal{R}_{q}$, and $e_{i}(x)$, drawn from a small error distribution, typically a discrete Gaussian, the RLWE problem is to determine the secret polynomial $s(x)$ given several samples $\left(a_{i}, a_{i} \cdot s+e_{i}\right)$. The choice of $N$ and $q$ determine the security level $\lambda$ of the RLWE problem. A security level of $\lambda$ bits indicates $\sim 2^{\lambda}$ operations are required to break the decryption, with typical values of $\lambda \in\{128,192,256\}$.

The presence of the noisy polynomials $e_{i}(x)$ yields noisy HE operations. Furthermore, the noise grows as additional HE operations are performed. Once the noise has reached a certain threshold, the homomorphism between encrypted and plaintext operations breaks down and decryption yields an inaccurate result. Noise growth is a fundamental difficulty in scaling use of HE in practice.

In 2009, Gentry discovered a bootstrapping procedure, which removes noise from the ciphertext. By applying bootstrapping to an LHE scheme, Gentry constructed the first fully homomorphic encryption (FHE) scheme, supporting an unlimited number of additions and multiplications [7]. Using polynomial approximations, it became possible to compute arbitrary functions on encrypted data. While initial implementations of bootstrapping could last several minutes for a single ciphertext, recent HE schemes [8], [9] operating on Boolean circuits, rather than arithmetic circuits, enable bootstrapping on the order of milliseconds. However, performing addition and multiplication in Boolean circuits requires several Boolean operations, creating additional overhead.

LHE schemes such as CKKS often employ the residue number system (RNS) representation to efficiently perform arithmetic on large integers. Using RNS representation, the coefficient modulus $q$ is factored into several primes, $q=$ $\prod_{i=0}^{L-1} q_{i}$ for some depth $L$. For convenience, we denote $q=$ $\left\{\left\lfloor\log _{2}\left(q_{0}\right)\right\rfloor,\left\lfloor\log _{2}\left(q_{1}\right)\right\rfloor, \ldots,\left\lfloor\log _{2}\left(q_{L-1}\right)\right\rfloor\right\}$, that is, $q$ as a list of bit-widths of each coefficient modulus. CKKS also uses approximate arithmetic, such that $\operatorname{Dec}\left(c_{1} \cdot c_{2}+c_{3}\right) \approx m_{1}$. $m_{2}+m_{3}$ for ciphertexts $c_{i}=\operatorname{Enc}\left(m_{i}\right)$. As a result, the RNS form of the CKKS scheme [10] features superior arithmetic circuit performance compared to the BFV scheme [11], [12].

\subsection{Intel $^{\circledR}$ Optane ${ }^{\mathrm{TM}}$ PMem}

Recently, in Q2 2019, Intel released a non-volatile, byteaddressable memory in the form of DIMMs, named Intel ${ }^{\circledR}$ Optane $^{\mathrm{TM}}$ Persistent Memory or Intel ${ }^{\circledR}$ Optane ${ }^{\mathrm{TM}}$ PMem ${ }^{1}$, which is compatible with $2^{\text {nd }}$ Generation Intel ${ }^{\circledR}$ Xeon ${ }^{\circledR}$ Scalable processors. This type of memory sits between memory and storage, delivering the best of two worlds through the convergence of memory and storage product traits. The persistent memory modules may coexist with traditional DDR4 DRAM DIMMs on the same platform, and run up to 2,666 MT/s while operating at 12 , 15 , or $18 \mathrm{~W}$. As of time of this writing, Intel offers persistent modules in sizes from 128 GB to 512 GB, which allows 2socket platforms to store up to $6 \mathrm{~TB}$ of data.

Systems equipping Optane PMem feature two operating modes: Memory Mode and App Direct Mode. Memory Mode aims at exposing a system with a huge volatile memory capacity while being completely transparent to applications and operating systems, and hence the persistence attribute is lost. In contrast, in App Direct mode, the software sees the DRAM and the persistent memory as two distinct memory pools and may choose what data to place into each tier.

In Memory Mode, DRAM is managed by the CPU memory controller as a direct-mapped write-back cache. Consequently, and as expected, data locality plays a relevant role in terms of performance, because access latency is the same as DRAM when an access hits, but it has to pay for

\footnotetext{
1. Formerly known as Intel ${ }^{\circledR}$ Optane ${ }^{\mathrm{TM}}$ DC Persistent Memory.
} 
the cost of accessing DRAM and persistent memory when the access misses in DRAM. The access to the Intel Optane PMem is optimized for 256 byte transfers and since the CPU requests data in chunks of 64 bytes, the DIMM controller integrates a media prefetch buffer of the recently accessed 256 bytes to rapidly respond to the CPU if data is already found in this buffer.

Compatible CPUs integrate new performance counters for the analysis of Intel Optane PMem-enabled software at different architectural levels [13]. The core counters are able to identify which data addresses have been referenced and which part of the memory system (including PMem) provided the data. The uncore counters provide information such as the traffic volume observed by the CPU memory controllers on the different memory types. The SMART counters may be used to determine the locality of the software media access patterns.

\section{Related Work}

Privacy-preserving machine learning (PPML) has gained interest in recent years. Given a machine learning model $M$, and input data $x$, typically owned by separate parties, one goal of PPML is to perform inference, i.e. compute $M(x)$, while reducing the data leakage among parties. Previous work typically uses one or more of several privacypreserving primitives, including secure multi-party computation (MPC), HE, differential privacy, and more recently, functional secret sharing [14]. Differential privacy seeks to minimize the leakage of $M$ or $x$ resulting from observing the result $M(x)$. In contrast, cryptographic techniques such as MPC and HE seek to minimize the data leakage during the computation process.

MPC-based DNN inference includes SecureML [15], XONN [16], SecureNN [17], ABY3 [18], PySyft [19], TFEncrypted |20|, and CrypTFlow [21]. HE-based DNN inference was introduced by the seminal CryptoNets paper [22], which performed inference on a 5-layer network on the MNIST dataset. Subsequent work has improved the performance, scaled to larger networks, and integrated with DNN compiler technologies [2], [23], [24], [25], [26]. While, due to DRAM limitations, the largest model reported to date running inference using HE is MobileNetV2 (a lightweight network with a small number of parameters) reduced by an expansion factor of 0.35 from the original size [23], we report results running MobileNetV2 on its largest expansion factor plus, for the first time in the literature, a fully-featured DNN: ResNet-50. Several works also combine multiple privacy-preserving primitives, such as HE with MPC [27], [28].

One difficulty in using HE for DNN inference is the choice of plaintext packing, which enables encoding of multiple scalars into a single plaintext. HE operations on the plaintext, as well as the encrypted ciphertext, apply to each scalar, in a single-instruction multiple data (SIMD) manner. Given a tensor of shape $N \times C \times H \times W$, batchaxis packing encodes the tensor as a $C \times H \times W$-ciphertext tensor, each storing $N$ scalars. Inter-axis packing uses a different encoding scheme, for instance as an $N$-ciphertext tensor, each storing $C \times H \times W$ scalars. In general, batchaxis packing attains high throughput at the cost of high latency and high total memory requirement, whereas interaxis packing attains low latency and memory usage at the cost of low throughput. Previous work has used both batchaxis packing [2], [22], [23] and inter-axis packing [27], [29], [30]. The choice of batch-axis packing requires hundreds of gigabytes of memory for MobileNetV2 [23], preventing scaling to larger networks on conventional DRAM systems.

A number of recent software efforts to develop and optimize HE primitives on top of different architectures exist [31], [32], [33], [34]; however, only a few very recent DNN frameworks are known supporting HE [2], [23], [26], [35] and these are tuned for DRAM-friendly access patterns. To date, no previous report exists of DNN inference leveraging HE on top of non-DRAM memory technology.

\section{Experimental Setup}

In this section, we describe the technical details of our experimental setup, including our testbed system, the inference engine we used, and the neural network models that served as our use cases.

\subsection{Testbed}

Our experiments are performed in a dual socket system using Intel ${ }^{\circledR}$ Xeon ${ }^{\circledR}$ Platinum $8260 \mathrm{~L}$ processors, with 24 cores on each socket running at a nominal frequency of $2.30 \mathrm{GHz}$. For all experiments involving persistent memory, twelve 512 GB Intel Optane PMem DIMMs were used. Each of these modules features a theoretical bandwidth of $7.3 \mathrm{~GB} / \mathrm{s}$ for read accesses and $2.4 \mathrm{~GB} / \mathrm{s}$ for write accesses [36]. For the Memory Mode (MM) experiments, we have worked with two different configurations. The first configuration (MM32) uses four 8 GB DRAM DIMMs, while the second (MM96) uses twelve DIMMs. For the DRAM-only experiments (DO), we have used twelve 16 GB DRAM DIMMs. Figure 1 shows an overview of the MM32, MM96 and DO configurations. Both DRAM DIMM models use DDR4 and feature a theoretical bandwidth of $21.3 \mathrm{~GB} / \mathrm{s}$.

Equipping reduced DRAM space and bandwidth on the MM32 configuration, we demonstrate the viability of our target use case on an energy-friendly memory configuration, being the energy consumption per byte of DRAM about 10 times higher than that of the Optane PMem (375 mW/GB vs. $35 \mathrm{~mW} / \mathrm{GB}$ ).

Our system runs Fedora 27 (Workstation Edition) with kernel version 4.18.8. All the software used was compiled from sources using GCC 7.3.1 targeting the native architecture.

\subsection{Inference Engine}

Intel ${ }^{\circledR}$ HE-Transformer for nGraph ${ }^{\mathrm{TM}}$ 23 is an HE backend to the Intel nGraph Compiler [37], a graph compiler and runtime for artificial neural networks. This backend supports the CKKS encryption scheme and relies on the simple encrypted arithmetic library (SEAL) [31] for the implementation. Operations that involve comparison (ReLU, MaxPool, etc.) are not supported natively in the CKKS scheme. To perform these operations, HE-Transformer uses a client-aided protocol in which the server sends the encrypted data to the client, which decrypts the data, performs 


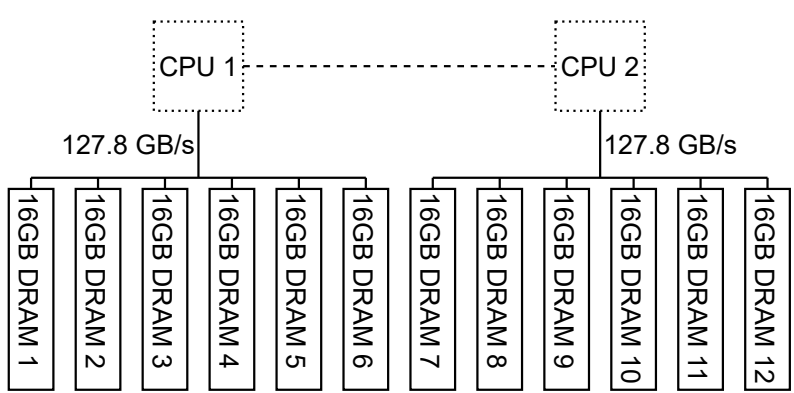

(a) DRAM-only, 192 GB DRAM (DO)

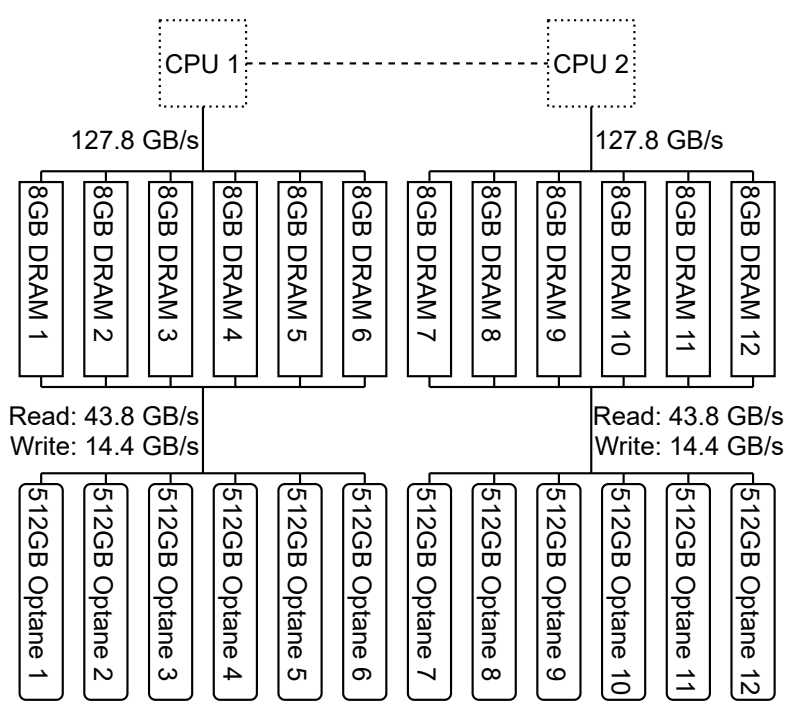

(b) Memory Mode, 96 GB DRAM + 6 TB Intel Optane (MM96)

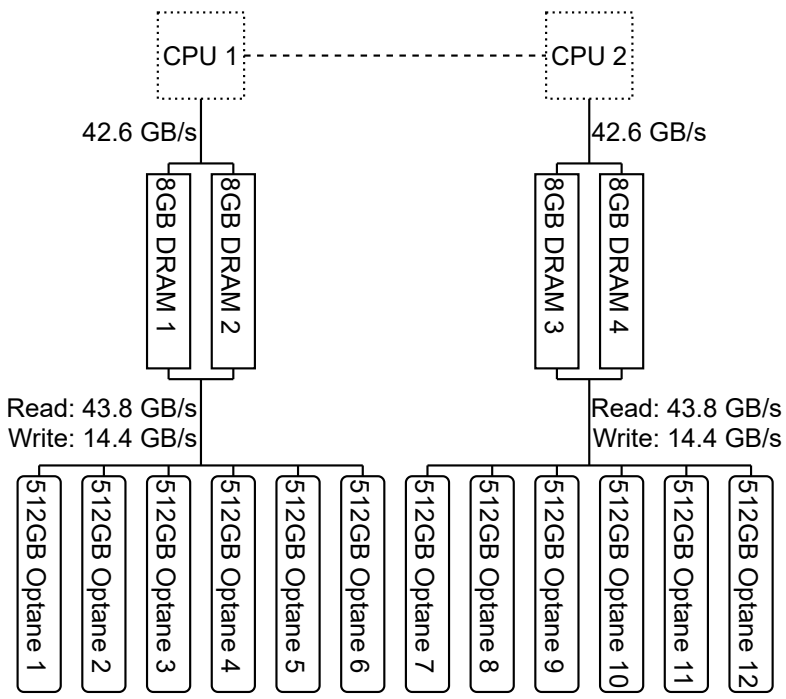

(c) Memory Mode, 32 GB DRAM + 6 TB Intel Optane (MM32)

Fig. 1. Logical view of the DO and MM memory configurations. the operation, encrypts the output, and sends it back to the server. This approach acts as a bootstrapping process to refresh the ciphertext noise, while also performing the comparison operation. However, it may leak the model weights to the client. This leakage may be mitigated using garbled circuits [28] but this approach currently suffers from an even higher runtime overhead, so we do not consider it in this work. HE-transformer enables data scientists to train networks on the hardware of their choice, then easily perform inference on encrypted data using popular deep learning frameworks such as TensorFlow.

In our experiments we have used HE-Transformer v0.6.0, which features the following dependencies: TensorFlow v1.14.0, nGraph v0.25.0, nGraph-bridge v.0.18.0, and SEAL v3.3.1.

We use the same encryption parameters as [23] in all of our experiments, namely:

- Polynomial modulus degree: $N=4096$

- Security level: $\lambda=128$

- Coefficient modulus: $q=\{30,22,22,30\}$

As in [23], we consider the use case of a plaintext model and encrypted data.

\subsection{Neural Network Models}

We make use of two well-known neural network models as our use cases: MobileNetV2 and ResNet-50.

\subsubsection{MobileNetV2}

MobileNetV2 [38] is a neural network model that uses depth-wise separable convolution to reduce the model size and complexity. Thanks to these layers, MobileNetV2 requires roughly 9 times less computation than comparable neural networks, making it ideal for use in mobile and embedded systems. This efficiency makes it particularly suitable for $\mathrm{HE}$, since the main disadvantage of this type of encryption is its high overhead. The MobileNetV2 architecture features two modifiable parameters:

- Width Multiplier: The number of channels in each layer with respect to the original model.

- Input Resolution: Size of width and height of the square image received by the network.

We denote a specific MobileNetV2 architecture with a tuple: (width multiplier, input resolution). The higher the parameter values, the greater the accuracy obtained but at the cost of larger memory footprint and longer computation time. Different configurations of both parameters have been tested in our experiments.

\subsubsection{ResNet-50}

Residual Network (ResNet) is a popular family of convolutional neural networks used for many computer vision applications [39]. These networks are easier to optimize than traditional DNNs, which translates into shorter training times. ResNet-50 is 50 layers deep and may classify images with a resolution of $224 \times 224$ pixels into 1,000 different categories. In the ImageNet dataset |40|, it attains a top 1 accuracy of $75 \%$ and a top 5 accuracy of $92 \%$. 


\subsection{Profiling Tools}

We have leveraged two profiling frameworks to confirm whether the application is using the PMem efficiently: Extrae [41]/Paraver [42], and Intel ${ }^{\circledR}$ VTune ${ }^{\mathrm{TM}}$ Platform Profiler [43]. Extrae is a library that monitors parallel applications (using OpenMP, MPI, and pthread, among others) and emits an activity record on a Paraver trace-file. Paraver is a post-mortem visualization tool for qualitative and quantitative analysis. Intel VTune Platform Profiler, on the other hand, is both the collector and visualizer for a system-wide profiling tool that allows obtaining a holistic view of system behavior including CPU, memory, network, and disk usage. While both tools collect PMem-related metrics through the CPU performance counters, they have some fundamental differences. Extrae/Paraver are targeted to explore a singleapplication performance by means of instrumentation and sampling, which enables a precise characterization, leading the resulting trace-file size to depend on the application activity. Intel VTune Platform Profiler samples system activity periodically, which allows monitoring longer runs independently of the application activity, but the tool does not attribute performance to specific routines.

\section{EXPERIMENTAL ANALYSIS}

In this section, we present and analyze the results of our indepth performance evaluation. We first focus our attention on the MobileNetV2 model. Next, we discuss the ResNet-50 network.

\subsection{MobileNetV2}

We have used the pre-trained MobileNetV2 models offered by TensorFlow ${ }^{2}$, which vary in the width multiplier $(0.35$, $0.5,0.75,1.0,1.3$, and 1.4) and input resolution $(96,128,160$, 192, and 224). For the two largest width multiplier settings only the 224 input resolution is available.

We performed experiments with all possible configurations to determine the memory consumption and the accuracy (top 1 and top 5) of each network when leveraging HE. We chose a batch size of 2,048 because it is the maximum possible value for the chosen encryption parameters. Figure 2, Figure 3, and Figure 4, show the results of these executions. As expected, both the memory footprint and the obtained accuracy increase with larger models. The largest model attains a top 1 accuracy of $75.1 \%$ and top 5 accuracy of $91.6 \%$, requiring $1.2 \mathrm{~TB}$ of memory. Large models have not been feasible to be run in discrete systems so far because of these memory requirements. Thanks to recently-emerged persistent memory technology, now we have the amount of memory required for this task; however, the feasibility of running inference on top of the added latency this technology poses remained to be seen.

We have obtained the run times as an average of ten repetitions. The maximum relative standard deviation is $3.2 \%$. Figure 5 shows the times with the MM32 configuration. The DO configuration has been used as a baseline, although only the six smallest models fit within 192 GB of RAM, starting at 71 GB. Table 1 shows the execution

2. https://github.com/tensorflow/models/tree/master/research/ slim/nets/mobilenet
TABLE 1

MobileNetV2 time comparison (MM vs. DO)

\begin{tabular}{crrrr}
\hline \multirow{2}{*}{ Model } & $\begin{array}{c}\text { Memory } \\
\text { Usage (GB) }\end{array}$ & $\begin{array}{c}\text { Time (s) } \\
\text { DO }\end{array}$ & $\begin{array}{c}\text { Time (s) } \\
\text { MM96 }\end{array}$ & $\begin{array}{r}\text { Time (s) } \\
\text { MM32 }\end{array}$ \\
\hline$(0.35,96)$ & 71 & 650 & 675 & 714 \\
$(0.35,128)$ & 125 & 1,158 & 1,183 & 1,262 \\
$(0.50,96)$ & 80 & 842 & 867 & 923 \\
$(0.50,128)$ & 144 & 1,503 & 1,537 & 1,653 \\
$(0.75,96)$ & 153 & 1,393 & 1,452 & 1,545 \\
$(1.00,96)$ & 157 & 1,621 & 1,691 & 1,833 \\
\hline
\end{tabular}

times of these models. The DO configuration (using $192 \mathrm{~GB}$ DRAM) is merely up to $4 \%$ faster than MM96 (using 96 GB DRAM as cache) and 11\% faster than MM32 (using 32 GB DRAM as cache), despite the considerable raw performance difference of the main memory subsystem leveraged by the latter with respect to DRAM. The MM32 configuration populates only 2 of the 6 available DRAM DIMMs, yielding a third of the bandwidth compared to the DO configuration, which is fully populated. In a memory-bandwidth-bound application, we would expect the execution times to be further impacted. However, the executions with the fully populated system are only about $10 \%$ faster, which indicates that memory bandwidth is not the main bottleneck, being a positive first indicator of the feasibility of leveraging persistent memory technologies as an enabler for large DNN inference with HE.

The additional latency incurred by the memory controller to manage the cache in MM is also part of this time difference. We have evaluated the latency incurred by the memory controller when in $\mathrm{MM}$ through Intel ${ }^{\circledR} \mathrm{MLC} \mathrm{C}^{\sqrt{3}}$, and observed that local and remote socket accesses experience $10 \%$ and $6 \%$ overhead, respectively.

\subsubsection{Analysis with Paraver}

Since HE-Transformer exploits parallelism through OpenMP [44] to deploy the most time-consuming parts of the library, we benefit from the Extrae abilities to automatically instrument this runtime to monitor the application behavior. We have additionally instrumented manually a number of functions of interest, such as: convolution, multiplication, reshape, etc. We correlate with Paraver the instrumented functions in HE-Transformer with several performance metrics, including CPU hardware counters. We have collected the following performance counters to analyze the performance of the Intel Optane PMem in Memory Mode:

- MEM_LOAD_RETIRED.LOCAL_PMM: Retired load instructions with local persistent memory as the data source and the data request missing L3 and DRAM cache.

- MEM_LOAD_L3_MISS_RETIRED.REMOTE_PMM: Retired load instructions with remote persistent memory as the data source and the data request missing L3 and DRAM cache.

3. https://software.intel.com/content/www/us/en/develop/ articles/intelr-memory-latency-checker.html 


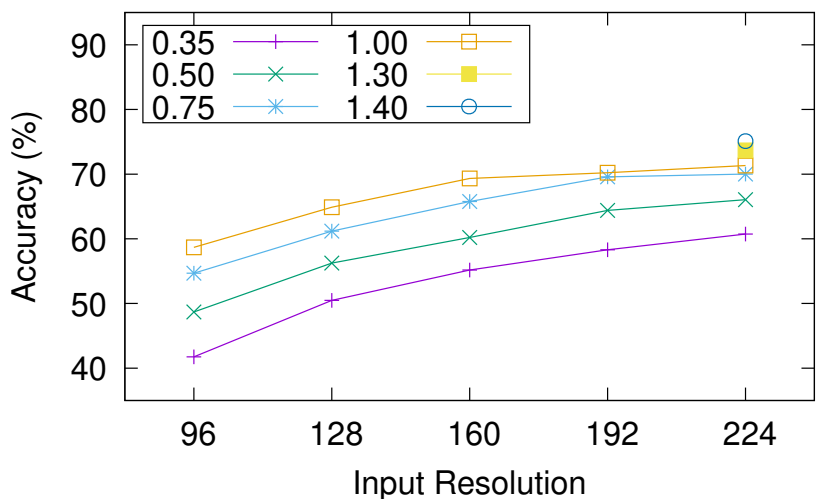

Fig. 2. Top 1 accuracy.

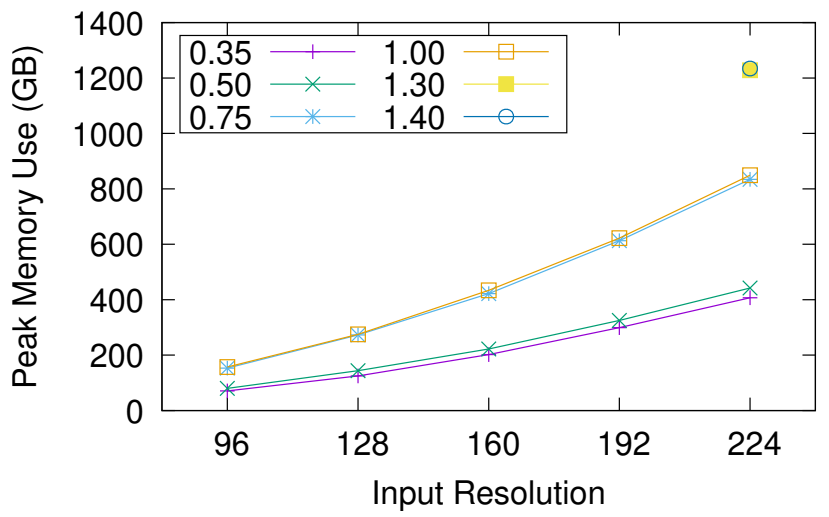

Fig. 4. Memory footprint.

- MEM_LOAD_L3_MISS_RETIRED.LOCAL_DRAM: Retired load instructions whose data sources missed L3 but were serviced from local DRAM.

- MEM_LOAD_L3_MISS_RETIRED.REMOTE_DRAM: Retired load instructions whose data sources missed L3 but were serviced from remote DRAM.

Despite using sampling mechanisms, the long running times of the largest models generate overwhelmingly large traces. For this reason, only three small models have been profiled in-depth for MobileNetV2: $(0.35,96),(0.35,128)$, and $(0.75,96)$.

Table 2 shows the result of the largest MobileNetV2 model profiled $(0.75,96)$ on the MM32 configuration. Since the values for the local and remote sockets were balanced, these are grouped in the table with a value that aggregates the number of accesses across the sockets. We have only explored the operations of the inference phase for the time measurement. As we will discuss in Section 5.1.2, there is an initialization phase at the beginning of the network execution in which HE-transformer executes a number of optimization passes. Consequently, we have decided to exclude this phase because in a production scenario it would be executed once, while the inference phase would be invoked multiple times.

The main difference between the smallest and largest models is the absolute number of accesses to DRAM and PMem spaces. The percentage of DRAM accesses for Convolution decreases slightly $(46 \%)$ in the smallest model while

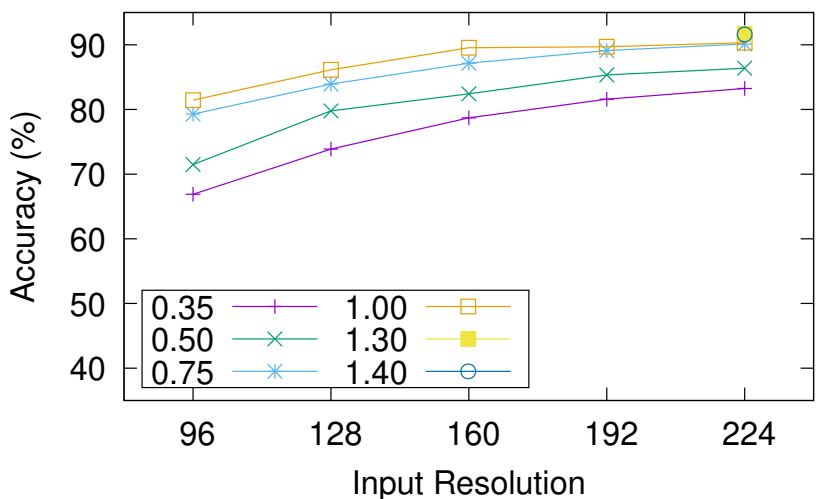

Fig. 3. Top 5 accuracy.

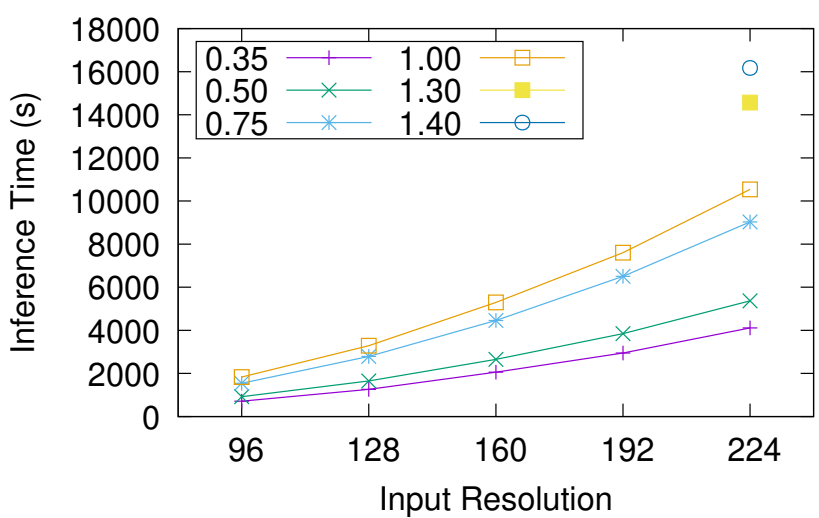

Fig. 5. Inference time (MM32).

the percentage for the BoundedRelu increases (49\%). The ratios of PMem accesses, the main focus of our study, are very similar to the smallest models using the same DRAM configuration.

The most time-consuming operation is Convolution, representing almost $92 \%$ of the execution, although it only accounts for $14 \%$ of the PMem accesses. Convolution only accesses PMem every $\sim 59$ DRAM accesses, which indicates that DRAM caches the working set efficiently and therefore the PMem is not limiting the execution time of this operation.

The second most time-consuming operation is BoundedRelu, representing $3.7 \%$ of the execution. This operation is not supported by the encryption scheme and in a production scenario it would be performed by a remote trusted machine using the client-aided protocol discussed in Section 4.2 . With the current setup, the operation is performed on the same machine, which has to: decrypt, operate, and encrypt the result. The encryption/decryption processes are strongly dependent on modified Fast Fourier Transform (FFT) operations, which feature non-sequential memory accesses. This explains the high number of accesses to PMem in BoundedRelu when compared to the remaining operations. To evaluate that this approach was not impacting the overall behavior of the application, we conducted an analysis of the operations immediately following the invocation of BoundedRelu. We observe that the values of cache misses in these operations are similar to those of the functions of the same type prior to a BoundedRelu. In a production 
TABLE 2

Extrae metrics running MobileNetV2 $(0.75,96)$ on the MM32 configuration

\begin{tabular}{l|rr|rr|rr|r}
\multicolumn{1}{c|}{ Function } & \multicolumn{2}{|c|}{ Time (s) } & DRAM (K-Loads) & PMem (K-Loads) & DRAM/PMem \\
\hline Add & 26,605 & $0.78 \%$ & 6,333 & $2.23 \%$ & 1,345 & $6.02 \%$ & 4.75 \\
AvgPool & 1,077 & $0.03 \%$ & 80 & $0.03 \%$ & 10 & $0.05 \%$ & 7.65 \\
BoundedRelu & 125,401 & $3.70 \%$ & 88,402 & $30.90 \%$ & 17,198 & $76.92 \%$ & 5.14 \\
Concat & 10,578 & $0.31 \%$ & 115 & $0.04 \%$ & 5 & $0.03 \%$ & 20.29 \\
Constant & 24,898 & $0.73 \%$ & 664 & $0.23 \%$ & 32 & $0.15 \%$ & 20.46 \\
Convolution & $3,113,888$ & $91.76 \%$ & 187,003 & $56.37 \%$ & 3,152 & $14.10 \%$ & 59.32 \\
Multiply & 9,652 & $0.28 \%$ & 1,841 & $0.64 \%$ & 489 & $2.19 \%$ & 3.76 \\
Reshape & 51,041 & $1.50 \%$ & 870 & $0.30 \%$ & 103 & $0.46 \%$ & 8.39 \\
Result & 40 & $0.00 \%$ & 117 & $0.04 \%$ & 0.5 & $0.00 \%$ & 207.42 \\
Slice & 30,338 & $0.89 \%$ & 576 & $0.20 \%$ & 19 & $0.09 \%$ & 30.11 \\
\hline Total & $3,393,517$ & & 286,066 & & 22,358 & & 12.79 \\
\hline
\end{tabular}

scenario, we expect this value to be slightly lower because not performing the operation would not invalidate as many cache entries as we are currently observing. However, there would be a higher latency in the unsupported operations because of the need to communicate the data over the network.

We also found that Add and Multiply operations experience a ratio between DRAM and PMem accesses below 5, which is notably low. However, these only account for $1 \%$ of the total time; hence, this low ratio does not significantly affect the overall execution.

\subsubsection{Analysis with Inte ${ }^{\circledR}$ VTune ${ }^{T M}$ Platform Profiler}

We used the Intel ${ }^{\circledR}$ VTune ${ }^{\mathrm{TM}}$ Platform Profiler to further confirm whether the workload used the underlying memory architecture efficiently. The tool provides independent performance reports for each socket (among other components) but for the sake of brevity we only show the profiling data from the socket that performs the application initialization.

Figure 6 depicts the metrics when the system runs the application and the results evidence two execution phases. The first phase corresponds to the initialization and optimization of the computation graph that describes the model. This phase executes in a single core, which relates to the low overall activity observed during this phase. The second phase corresponds to the inference, which in contrast to the initialization, runs mostly in parallel on all processor cores. In the inference phase, and according to the profiling results, the workload is fairly balanced among the sockets. We present the results for the MobileNetV2 $(1.4,224)$ architecture, which are highly similar to those of the smaller models.

Figure 6 a shows that the inference phase starts a behavior corresponding to mostly sequential accesses. As we described earlier, Intel Optane PMem is optimized for 256-byte media operations and the PMem modules feature a media prefetch buffer that stores contiguous bytes. In sequential access patterns, we expect a 0.75 hit ratio because the first data access causes a miss in the prefetch buffer but the three subsequent accesses hit on the buffer. Again, the sequential access pattern exposed by the workload favors the memory accesses and matches with the metrics reported by Paraver. Sequential access patterns are justified in further detail in Section 5.3

To assess whether the persistent memory implementation poses a bandwidth limitation, we have used the MM32 configuration. In this situation, each DRAM DIMM features an individual bandwidth circa $21 \mathrm{~GB} / \mathrm{s}$ and since there are two DIMMs per socket populated, the bandwidth per socket is close to $42 \mathrm{~GB} / \mathrm{s}$ (see Figure 1c). Our profiling reveals that the average memory bandwidth in one socket is $2.02 \mathrm{~GB} / \mathrm{s}$ for reading and and $2.15 \mathrm{~GB} / \mathrm{s}$ for writing with peaks of around $20 \mathrm{~GB} / \mathrm{s}$ (Figure 6b). A similar behavior is observed in the sibling socket (but not shown in the figure). Each PMem module, on the other hand, yields an individual bandwidth of $7.3 \mathrm{~GB} / \mathrm{s}$ for reading and $2.4 \mathrm{~GB} / \mathrm{s}$ for writing and since each socket is fully populated with six DIMMs, the bandwidth per socket is close to $43.8 \mathrm{~GB} / \mathrm{s}$ for reading and $14.4 \mathrm{~GB} / \mathrm{s}$ for writing. Figure $6 \mathrm{c}$ illustrates that neither writing nor reading bandwidth attain the maximum value.

These results are aligned with those exposed in Section 5.1.1 and reinforce our statement that Intel Optane PMem yields efficient executions for this type of workloads.

\subsection{ResNet-50}

With our setup, we have been able to perform, for the first time, inference using the ResNet-50 model, hence becoming the largest neural network ever run using HE. Leveraging a batch of 2,048 (the maximum value possible using the encryption parameters mentioned in Section 4.2 the complete execution lasts over 63 hours. Figure 7 shows the behavior of the CPU throughout the execution. We observe the alternation of parallel functions (which use the 48 available cores) with sequential functions, following the expected fork-join model. Since we are leveraging the use case of plaintext models (and encrypted data), despite ResNet- 50 being much more computationally demanding than the considerably smaller MobileNetV2 $(1.4,224)$ model, the former requires a slightly smaller amount of memory than the latter. Figure 8 shows that the ResNet-50 peak memory consumption stands below $900 \mathrm{~GB}$.

Due to the length of the execution, it has been impossible for us to profile it with any of the the tools at our reach. Since the predominant function in this type of networks is convolution, and the access pattern for this function remains invariant with the input size, we reasonably postulate that ResNet-50 leverages our persistent-memory-based memory setup as efficiently as MobileNetV2. This postulate applies to any other convolution-dominated model. 


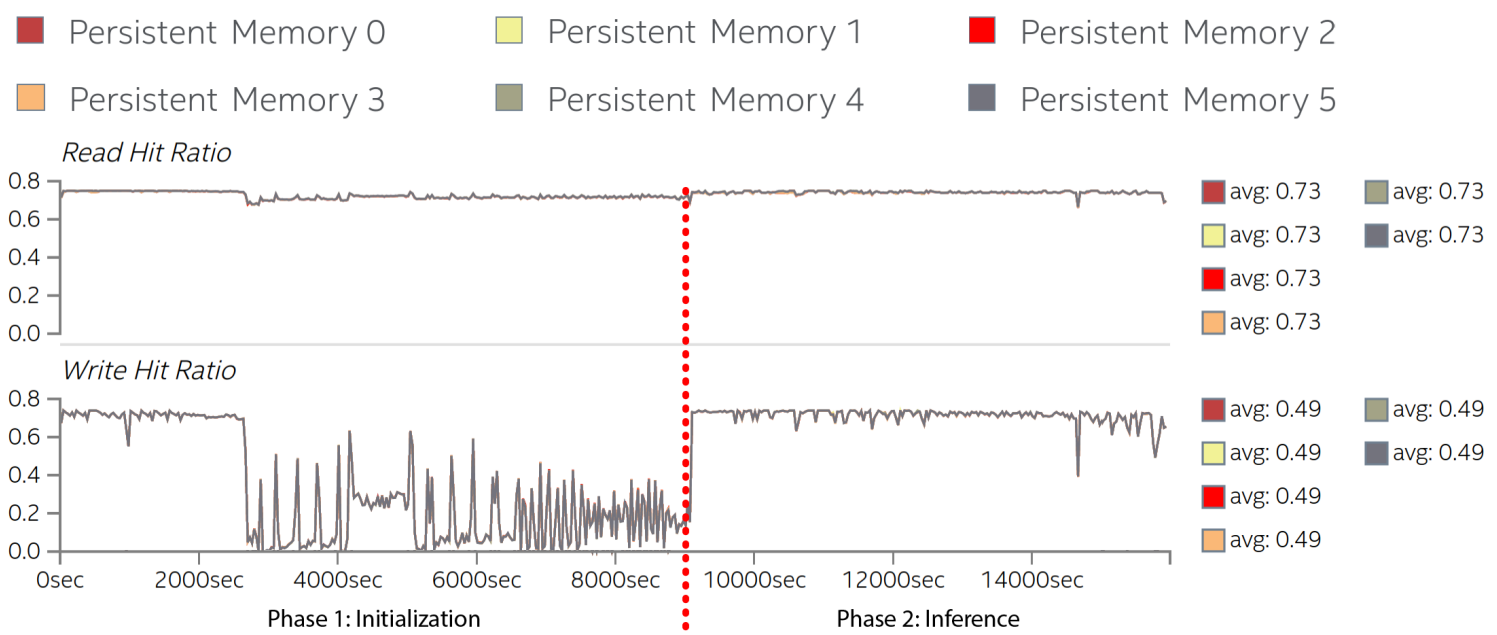

(a) Optane cache hit ratio
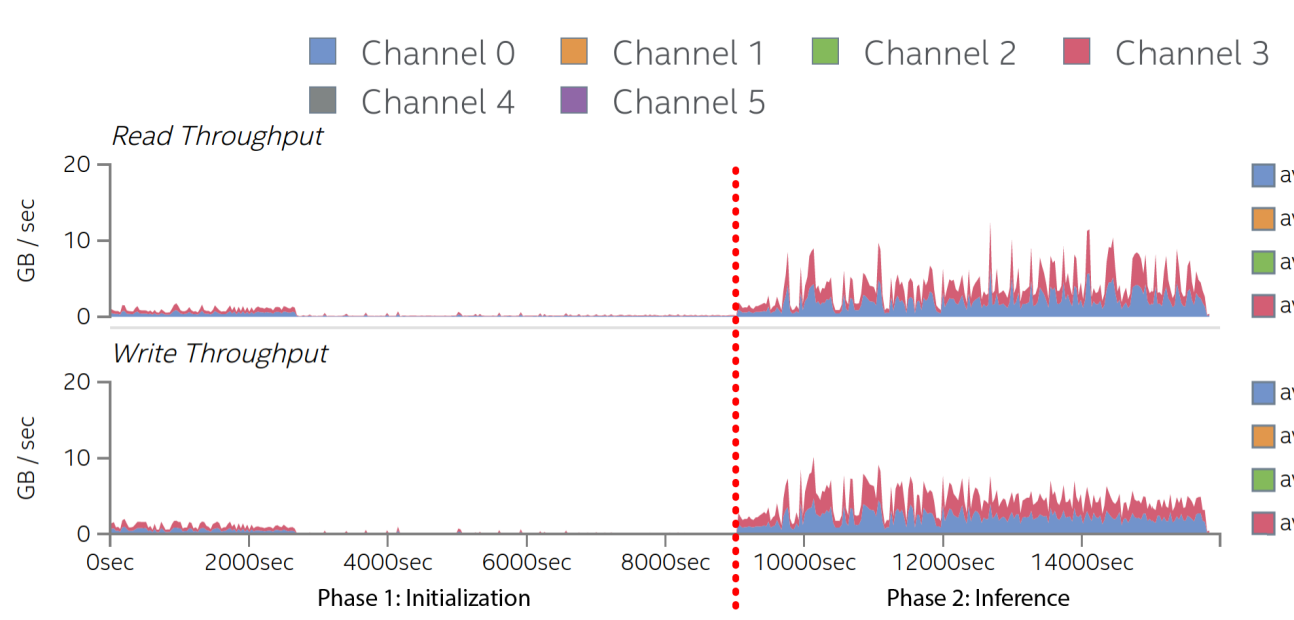

(b) DRAM traffic

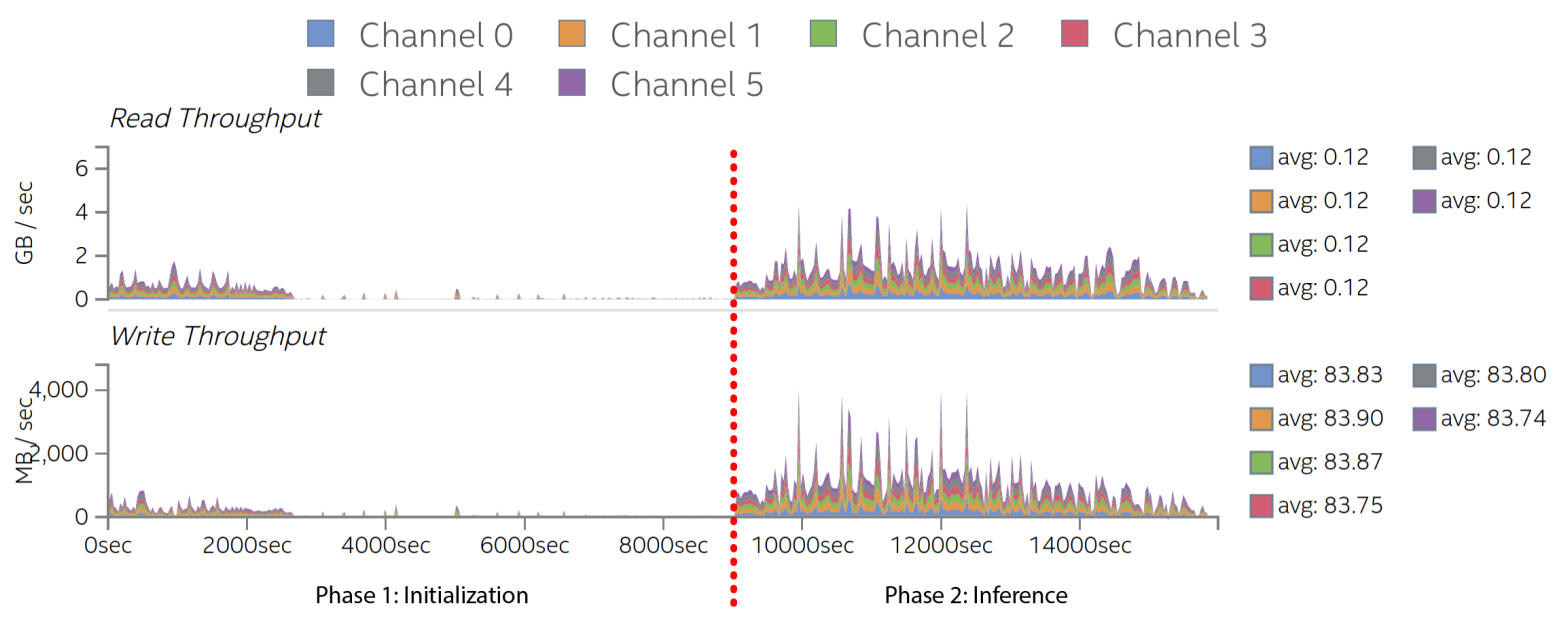

(c) PMem traffic

Fig. 6. Platform Profiler relevant data for MobileNetV2 $(1.4,224)$ in MM32. 


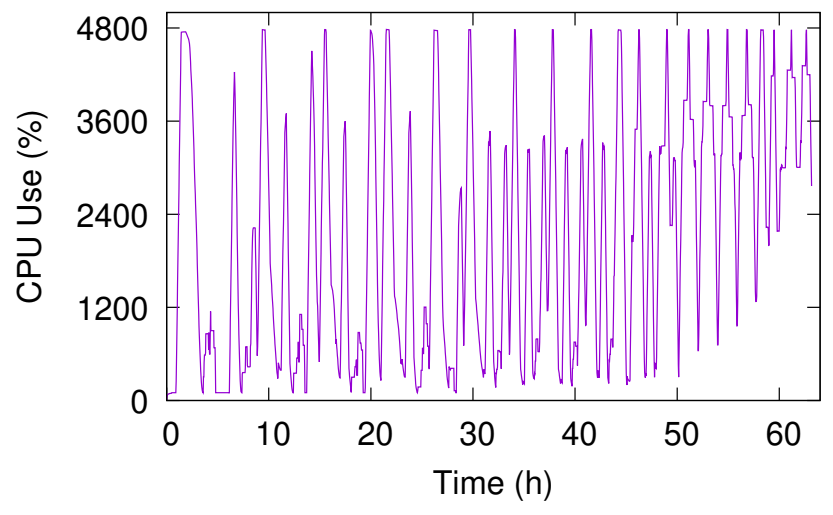

Fig. 7. ResNet-50 CPU usage.

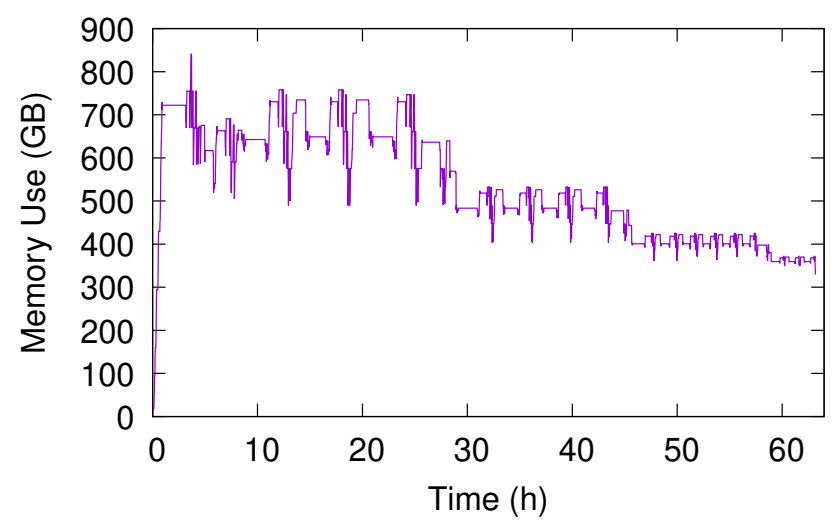

Fig. 8. ResNet-50 memory use.

\subsection{Discussion: Access Sequentiality}

Sections 5.1 and 5.2 show Intel Optane PMem yielding execution efficiency for large HE models, due to cachefriendly sequential memory access patterns, particularly in the Convolution operation. The Convolution operation is dominated by ciphertext-plaintext addition and ciphertextplaintext multiplication, each of which features sequential memory accesses, implemented in SEAL [31]. Note that we do not consider the CKKS rescaling operation here, since its runtime impact is minimized by the use of lazy rescaling [23|. SEAL represents a ciphertext in RNS form as a $2 \times L \times N$-length vector of unsigned 64-bit integers. However, ciphertext-plaintext operations require only one of the two ciphertext polynomials. The plaintext argument in SEAL is represented as an $L \times N$-sized vector of unsigned 64-bit integers. We use the optimization from nGraphHE2 [23], in the case where the plaintext encodes a single scalar, such that the plaintext argument is an $L$-length vector.

\subsubsection{Ciphertext-Plaintext Addition}

CKKS ciphertext-plaintext addition in RNS form requires element-wise addition followed by modular reduction of two polynomials in which each sum is reduced with respect to the coefficient modulus $q_{\ell}$.

The following algorithm shows the pseudocode for ciphertext-plaintext scalar addition. The ciphertext argument is arranged in memory such that the two-dimensional memory accesses are sequential.
1: function AdD CiPHeR-Plain $\operatorname{Scalar}\left(c \in \mathbb{Z}^{L \times N}, p \in\right.$ $\mathbb{Z}^{L}, q \in \mathbb{Z}^{L}$ )

2: $\quad$ for $\ell=1$ to $L$ do

3: $\quad t m p \leftarrow p[\ell]$

4: $\quad$ for $n=1$ to $N$ do

5: $\quad c[\ell][n] \leftarrow(c[\ell][n]+t m p) \bmod q[\ell]$

6: $\quad$ end for

7: $\quad$ end for

8: end function

\subsubsection{Ciphertext-Plaintext Multiplication}

CKKS ciphertext-plaintext multiplication in RNS form requires element-wise multiplication followed by modular reduction of two polynomials in which each product is reduced with respect to the coefficient modulus $q_{\ell}$. As in SEAL [31], nGraph-HE2 [23] uses Barrett reduction for efficient modulus reduction. The following algorithm shows the pseudocode for ciphertext-plaintext scalar multiplication, in the case where the coefficient modulus is less than 32 bits, as in our setting. The ciphertext argument is arranged in memory such that the two-dimensional memory accesses are sequential.

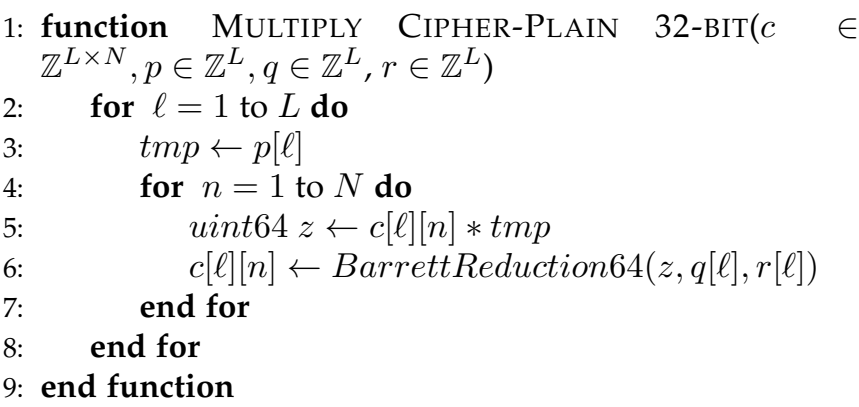

These algorithms show that the ciphertext accesses are sequential in memory. This yields the high ratio of cache hits seen in Figure 6a and supports the high efficiency experienced using Intel Optane PMem.

\section{Conclusions}

In this article we report running inference for the largest DNN models to date leveraging HE. For the first time in the literature, we use recently-emerged persistent memory technology as an enabler for such large memory footprints. Our novel analysis reveals that DNN inference leveraging HE features memory access patterns that yield efficient use of the Intel Optane PMem in Memory Mode. Sequential data accesses in the most common operations enable the accessed data to be efficiently cached in the DRAM, but also to make efficient use of the hardware prefetch buffers in the PMem. We propose a memory configuration with reduced DRAM size that is cost-efficient, equipping $1 / 3$ of the DRAM bandwidth while reducing merely $10 \%$ performance with respect to a fully-populated system.

\section{ACKNOWLEDGMENTS}

We would like to thank Jesus Labarta from BSC and Steve Scargall from Intel for their insightful and productive comments. 


\section{REFERENCES}

[1] M. Chase, H. Chen, J. Ding, S. Goldwasser, S. Gorbunov, J. Hoffstein, K. Lauter, S. Lokam, D. Moody, T. Morrison et al., "Security of homomorphic encryption," Tech. Rep., 2017. [Online] Available: HomomorphicEncryption.org

[2] F. Boemer, Y. Lao, R. Cammarota, and C. Wierzynski, "nGraphHE: A graph compiler for deep learning on homomorphically encrypted data," in Proceedings of the 16th ACM International Conference on Computing Frontiers, 2019, pp. 3-13.

[3] "Intel(R) Optane(TM) Persistent Memory," https://www. intel.com/content/www/us/en/architecture-and-technology/ optane-dc-persistent-memory.html 2020.

[4] R. L. Rivest, L. Adleman, M. L. Dertouzos et al., "On data banks and privacy homomorphisms," Foundations of Secure Computation, vol. 4, no. 11, pp. 169-180, 1978.

[5] J. H. Cheon, A. Kim, M. Kim, and Y. Song, "Homomorphic encryption for arithmetic of approximate numbers," in International Conference on the Theory and Application of Cryptology and Information Security. Springer, 2017, pp. 409-437.

[6] V. Lyubashevsky, C. Peikert, and O. Regev, "On ideal lattices and learning with errors over rings," in Annual International Conference on the Theory and Applications of Cryptographic Techniques. Springer, 2010, pp. 1-23.

[7] C. Gentry, "A fully homomorphic encryption scheme," Ph.D. dissertation, Stanford University, 2009.

[8] L. Ducas and D. Micciancio, "FHEW: Bootstrapping homomorphic encryption in less than a second," in Annual International Conference on the Theory and Applications of Cryptographic Techniques. Springer, 2015, pp. 617-640.

[9] I. Chillotti, N. Gama, M. Georgieva, and M. Izabachène, "Faster packed homomorphic operations and efficient circuit bootstrapping for TFHE," in International Conference on the Theory and Application of Cryptology and Information Security. Springer, 2017, pp. 377-408.

[10] J. H. Cheon, K. Han, A. Kim, M. Kim, and Y. Song, “A full RNS variant of approximate homomorphic encryption," in International Conference on Selected Areas in Cryptography. Springer, 2018, pp. 347-368.

[11] Z. Brakerski, "Fully homomorphic encryption without modulus switching from classical GapSVP," in Annual Cryptology Conference. Springer, 2012, pp. 868-886.

[12] J. Fan and F. Vercauteren, "Somewhat practical fully homomorphic encryption," IACR Cryptol. ePrint Arch., vol. 2012, p. 144, 2012.

[13] "perfmon for CLX processors," https://download.01.org/ perfmon/CLX 2020.

[14] T. Ryffel, D. Pointcheval, and F. Bach, "ARIANN: Low-interaction privacy-preserving deep learning via function secret sharing," in 34th Conference on Neural Information Processing Systems (NeurIPS), Vancouver, Canada, 2020

[15] P. Mohassel and Y. Zhang, "SecureML: A system for scalable privacy-preserving machine learning," in IEEE Symposium on Security and Privacy (SP). IEEE, 2017, pp. 19-38.

[16] M. S. Riazi, M. Samragh, H. Chen, K. Laine, K. Lauter, and F. Koushanfar, "XONN: XNOR-based oblivious deep neural network inference," in 28th USENIX Security Symposium (USENIX Security 19), 2019, pp. 1501-1518.

[17] S. Wagh, D. Gupta, and N. Chandran, "SecureNN: Efficient and private neural network training," IACR Cryptol. ePrint Arch., vol. 2018, p. 442, 2018.

[18] P. Mohassel and P. Rindal, "ABY3: A mixed protocol framework for machine learning," in Proceedings of the 2018 ACM SIGSAC Conference on Computer and Communications Security, 2018, pp. 3552.

[19] T. Ryffel, A. Trask, M. Dahl, B. Wagner, J. Mancuso, D. Rueckert, and J. Passerat-Palmbach, "A generic framework for privacy preserving deep learning," in Privacy Preserving Machine Learning (NeurIP Workshop), 2018.

[20] M. Dahl, J. Mancuso, Y. Dupis, B. Decoste, M. Giraud, I. Livingstone, J. Patriquin, and G. Uhma, "Private machine learning in TensorFlow using secure computation," 2018. [Online]. Available: https://arxiv.org/abs/1810.08130

[21] N. Kumar, M. Rathee, N. Chandran, D. Gupta, A. Rastogi, and R. Sharma, "CrypTFlow: Secure TensorFlow inference," in 41st IEEE Symposium on Security and Privacy, 2020.

[22] R. Gilad-Bachrach, N. Dowlin, K. Laine, K. Lauter, M. Naehrig, and J. Wernsing, "CryptoNets: Applying neural networks to en- crypted data with high throughput and accuracy," in International Conference on Machine Learning, 2016, pp. 201-210.

[23] F. Boemer, A. Costache, R. Cammarota, and C. Wierzynski, "nGraph-HE2: A high-throughput framework for neural network inference on encrypted data," in Proceedings of the 7th ACM Workshop on Encrypted Computing \& Applied Homomorphic Cryptography, 2019, pp. 45-56.

[24] E. Hesamifard, H. Takabi, and M. Ghasemi, "CryptoDL: Deep neural networks over encrypted data," 2017. [Online]. Available: https://arxiv.org/abs/1711.05189

[25] C. Boura, N. Gama, and M. Georgieva, "Chimera: A unified framework for B/FV, TFHE and HEAAN fully homomorphic encryption and predictions for deep learning," IACR Cryptol. ePrint Arch., vol. 2018, p. 758, 2018.

[26] R. Dathathri, O. Saarikivi, H. Chen, K. Laine, K. Lauter, S. Maleki, M. Musuvathi, and T. Mytkowicz, "CHET: An optimizing compiler for fully-homomorphic neural-network inferencing," in Proceedings of the 40th ACM SIGPLAN Conference on Programming Language Design and Implementation, 2019, pp. 142-156.

[27] C. Juvekar, V. Vaikuntanathan, and A. Chandrakasan, "GAZELLE: A low latency framework for secure neural network inference," in 27th USENIX Security Symposium (USENIX Security 18), 2018, pp. 1651-1669.

[28] F. Boemer, R. Cammarota, D. Demmler, T. Schneider, and H. Yalame, "MP2ML: A mixed-protocol machine learning framework for private inference," in International Conference on Availability, Reliability and Security (ARES), 2020.

[29] C. Jin, A. Al Badawi, J. L. Balagopal Unnikrishnan, C. F. Mun, J. M. Brown, J. P. Campbell, M. Chiang, J. Kalpathy-Cramer, V. R. Chandrasekhar, P. Krishnaswamy et al., "CareNets: Efficient homomorphic CNN for high resolution images," in NeurIPS Workshop on Privacy in Machine Learning (PriML), 2019.

[30] A. Brutzkus, R. Gilad-Bachrach, and O. Elisha, "Low latency privacy preserving inference," in Proceedings of the 36th International Conference on Machine Learning, ser. Proceedings of Machine Learning Research, vol. 97, Long Beach, California, USA, Jun. 2019, pp. 812-821. [Online]. Available: http: //proceedings.mlr.press/v97/brutzkus19a.html

[31] "Microsoft SEAL (release 3.3)," https://github.com/Microsoft/ SEAL Jun. 2019, Microsoft Research, Redmond, WA.

[32] W. Dai and B. Sunar, "cuHE: A homomorphic encryption accelerator library," in International Conference on Cryptography and Information Security in the Balkans. Springer, 2015, pp. 169-186.

[33] E. Chou, J. Beal, D. Levy, S. Yeung, A. Haque, and L. Fei-Fei, "Faster cryptonets: Leveraging sparsity for realworld encrypted inference," 2018. [Online]. Available: https: //arxiv.org/abs/1811.09953

[34] S. S. Roy, F. Turan, K. Jarvinen, F. Vercauteren, and I. Verbauwhede, "FPGA-based high-performance parallel architecture for homomorphic computing on encrypted data," in 2019 IEEE International Symposium on High Performance Computer Architecture (HPCA). IEEE, 2019, pp. 387-398.

[35] T. van Elsloo, G. Patrini, and H. Ivey-Law, "Sealion: A framework for neural network inference on encrypted data," in Proceedings of the 36th International Conference on Machine Learning, Long Beach, CA, 2019.

[36] "Analyzing the performance of Intel Optane DC Persistent Memory in App Direct mode in Lenovo ThinkSystem servers," https: //lenovopress.com/lp1083.pdf 2020.

[37] S. Cyphers, A. K. Bansal, A. Bhiwandiwalla, J. Bobba, M. Brookhart, A. Chakraborty, W. Constable, C. Convey, L. Cook O. Kanawi, R. Kimball, J. Knight, N. Korovaiko, V. Kumar, Y. Lao, C. R. Lishka, J. Menon, J. Myers, S. A. Narayana, A. Procter, and T. J. Webb, "Intel nGraph: An intermediate representation, compiler, and executor for deep learning," 2018.

[38] M. Sandler, A. Howard, M. Zhu, A. Zhmoginov, and L.-C. Chen, "MobileNetV2: Inverted residuals and linear bottlenecks," 2018.

[39] K. He, X. Zhang, S. Ren, and J. Sun, "Deep residual learning for image recognition," in Proceedings of the IEEE Conference on Computer Vision and Pattern Recognition, 2016, pp. 770-778.

[40] J. Deng, W. Dong, R. Socher, L.-J. Li, K. Li, and L. Fei-Fei, "ImageNet: A large-scale hierarchical image database," in IEEE Conference on Computer Vision and Pattern Recognition, 2009, pp. 248-255.

[41] "BSC Extrae," https://tools.bsc.es/extrae, 2020.

[42] "BSC Paraver," https://tools.bsc.es/paraver 2020. 
[43] "Intel(R) VTune(TM) Platform Profiler," https://software. intel.com/content/www/us/en/develop/documentation/ vtune-help/top/analyze-performance/plattorm-analysis-group/ platform-protiler-analysis.html 2020.

[44] L. Dagum and R. Menon, "OpenMP: An industry standard API for shared-memory programming," Computational Science $\mathcal{E}$ Engineering, IEEE, vol. 5, no. 1, pp. 46-55, 1998.

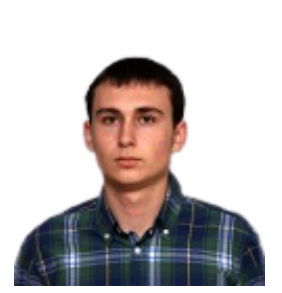

Guillermo Lloret-Talavera is a Jr. Research Engineer at the Barcelona Supercomputing Center (BSC), working in the Accelerators and Communications for HPC team. His interests include performance tuning for heterogeneous memory systems and deep learning frameworks.

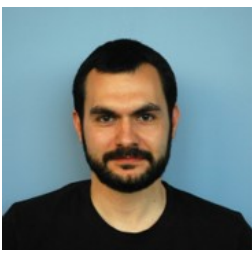

Marc Jorda is a Research Engineer at the Barcelona Supercomputing Center (BSC), working in the Accelerators and Communications for HPC team. His interests include performance tuning for heterogeneous memory systems, GPU-enabled applications, and deep learning frameworks.

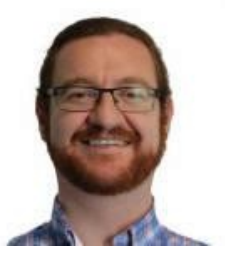

Dr. Harald Servat is an HPC system enthusiast with strong knowledge in monitoring systems, parallel programming models, compilers and computer architecture. He currently works at Intel Corp. on code modernization topics for the next generation HPC systems. Before that, he was the maintainer of the instrumentation library for the BSC performance tools suite (Extrae) while adapting it to new technologies and pursuing large scalability. In 2015, he received his Ph.D. in providing instantaneous metrics combining coarse-grain instrumentation and sampling techniques. During his research, he explored the performance of several in-production applications and applied code transformations to increase the application performance.

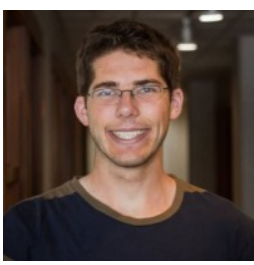

Fabian Boemer is a research scientist at Intel Corporation. He received his Master's degree from Stanford University in Computational and Mathematical Engineering in 2018. Fabian's interests lie in privacy-preserving machine learning, in particular homomorphic encryption (HE). Fabian maintains the nGraph-HE software library (ngra.ph/he), which enables deep learning on homomorphically encrypted data.

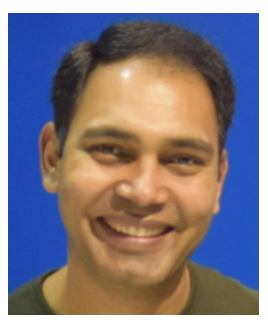

Chetan Chauhan is Optane Component Architect in Intel's Nonvolatile Memory Storage Group. His focus is on developing/simulating power and performance models for pathfinding new system architecture for Optane. He is very interested in exploring how Optane can help Al use cases like homomorphic encryption. He has received his Master's in Computer Engineering from Syracuse University.

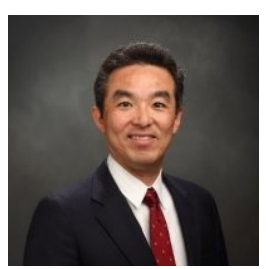

Dr. Shigeki Tomishima received his B.S. and M.S. degrees in Solid State Physics and his $\mathrm{Ph} . \mathrm{D}$. degree in Electric Engineering from Osaka University, Osaka, Japan in 1988, 1990 and 2002, respectively. After 20 years of DRAM memory array and architecture research in DRAM industry, he joined Intel Corporation to work on the embedded DRAM project. In 2014 he joined Intel Labs/Memory Architecture Lab to start working on advanced memory architecture research for future computing systems, the emerging memory technology including Compute-Near-Memory and Compute-In-Memory concepts. He has authored and coauthored more than 15 international conference papers, 10 journal papers, 15 invited talks, and holds 129 issued U.S. patents. He received Best Paper Reviewer Award from IEEE CAS in 2016 and serves as TPC member on A-SSCC, VLSI-DAT, ISQED, and as a paper reviewer on JSSC, SSC-L, TVLSI, MICRO, ISCA, AICAS, and ISCAS.

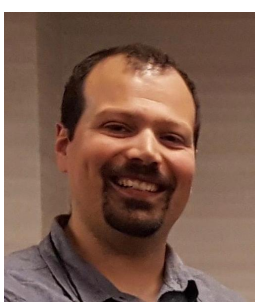

Nilesh N. Shah directs Computational Storage at Intel's Nonvolatile Memory Storage Group's Data Center division and likes hacking privacy preserving machine learning code for fun. $\mathrm{He}$ is specially interested in homomorphic encryption, federated deep learning and mixing and matching storage technologies with heterogeneous accelerators.

Dr. Antonio J. Peña holds a BS+MS degree in Computer Engineering (2006), and MS and PhD degrees in Advanced Computer Systems (2010, 2013), from Universitat Jaume I de Castelló, Spain. He is currently a Sr. Researcher at the Barcelona Supercomputing Center (BSC), where he leads the "Accelerators and Communications for HPC" team. Antonio is a former Marie Sklodowska-Curie Fellow and Juan de la Cierva Fellow. He is a recipient of the 2017 IEEE TCHPC Award for Excellence for Early Career Researchers in HPC. His research interests in the area of runtime systems and programming models for HPC include resource heterogeneity and communications. 